\title{
EVALUACIÓN DE LA ESTABILIDAD DE UN DIGESTOR ANAEROBIO TERMÓFILO
}

\author{
Edson Morales $\mathrm{Ch}^{* \mathrm{a}}$
}

\begin{abstract}
RESUMEN
Este estudio investiga los efectos de la reducción de tiempos de retención hidráulica, para evaluar la estabilidad de un digestor anaeróbico termófilo $\left(55^{\circ} \mathrm{C}\right)$, para tratar un lodo producido en una planta de tratamiento de aguas residuales de Valladolid, con una composición promedio de DQO 35,982 mg / L; SV 18,5 g / L; pH 8,08; N-NH3 347,5 mg / L; N-NKT $1833,8 \mathrm{mg} / \mathrm{kg}$.

Se propuso trabajar modificando el tiempo de retención hidráulica, reduciendo progresivamente de 22,52 a 4,5 días en tres momentos por un periodo de 1001 días, aumentando la velocidad de flujo de alimentación y el aumento en los valores de la velocidad de carga orgánica. Los resultados mostraron que el reactor entró a procesos de estrés por el aumento en la carga del reactor, pero fueron tolerados y continuó generando biogás y la eliminación de SV del 37 \% en promedio.
\end{abstract}

Palabras clave: digestión anaerobia, reactor termófilo, ácidos grasos volátiles

\section{STABILITY OF A THERMOPHILIC ANAEROBIO DIGESTOR UNDER NON-COMMON OPERATING CONDITIONS}

\begin{abstract}
This study investigates the effects of reducing hydraulic retention times, to assess the stability of a thermophilic anaerobic digester $\left(55^{\circ} \mathrm{C}\right)$, for treating a sludge produced in a treatment plant wastewater Valladolid, with a composition average COD 35,982 mg / L; SV 18,5 g / L; pH 8,08; N-NH3 347,5 mg / L; N-NKT 1833,8 mg / kg.

It was proposed to work modifying the hydraulic retention time progressively reducing from 22,52 to 4,5 days in three stages for a period of 1001 days, increasing the feed flow rate and increasing the values of the organic loading rate. The results showed that the reactor entered into stress processes due to the increase in the reactor load, but they were tolerated and continued generating biogas and the elimination of SV of $37 \%$ on average.
\end{abstract}

Key words: anaerobic digestion, thermophilic reactor, volatile fatty acids

a Investigador UVA, Departamento de Ingeniería Química Escuela de Ingeniería Industriales, Universidad de Valladolid, Valladolid - España. Email.: edson.morales.ch@gmail.com. 


\section{INTRODUCCIÓN}

Los procesos de fabricación, transformación, utilización, consumo y/o limpieza en las actividades humanas, generan residuos sólidos, emisiones y efluentes, los cuales van a generar un impacto negativo al medio ambiente. La falta de tratamiento y el no reaprovechamiento de los residuos es un problema que cada vez toma mayor protagonismo. Asimismo, la mala disposición final de las aguas residuales urbanas en los cuerpos receptores, contienen patógenos y coliformes lo cual lo convierte en una fuente de transmisión de enfermedades infectocontagiosas ${ }^{1}$.

En la actualidad existe una tendencia de desarrollo orientada hacia el ciclo urbano del agua para ser auto sostenible y que minimice el requerimiento de energía y la aportación de residuos al medio natural ${ }^{2}$. Por ello, es importante el tratamiento de aguas residuales, ya que el agua es un recurso de gran importancia para los seres vivos y se encuentra cada vez más escasa, por lo que se debe generar conciencia para cuidarla y reusarla adecuadamente ${ }^{3}$. Es importante regresar el agua que se toma de la naturaleza, en una condición "aceptable", para que siga su camino dentro del ciclo hidrológico ${ }^{4}$.

En la actualidad, las sociedades industrializadas deben asumir compromisos para el cuidado del medio ambiente, como por ejemplo la depuración de las aguas residuales; pero estos tratamientos también generan problemas ambientales como la generación de lodos, que están asociados a su tasa de crecimiento.

En la actualidad, nuestro país cuenta con una normativa para Plantas de Tratamiento de Aguas Residuales (PTAR), la cual regula los Límites Máximos Admisibles (LMP) . De este modo, tratar los vertimientos se convierte en una necesidad para mitigar la contaminación y preservar la biodiversidad que se encuentra en los cuerpos de agua.

En España, el tratamiento de los lodos que se producen en las Estaciones Depuradoras de Aguas Residuales (EDAR), se rigen por la ley $22 / 2011^{6}$. y la normativa específica (RD $1310 / 1990)^{7}$. Para la disposición y aplicación de los lodos de depuradora en los suelos agrícolas es regulada por la Directiva $86 / 278 / \mathrm{CEE}^{8}$, la cual promueve la protección del medio ambiente, en particular de los suelos. En el Perú, los lodos de las PTAR son manejados como residuos no peligrosos, salvo en los casos que el Ministerio de Vivienda, Construcción y Saneamiento determine lo contrario?.

El tratamiento anaerobio por Metanogénesis es muy utilizado en la estabilización de lodos de aguas residuales y residuos sólidos urbanos ${ }^{10}$. por la facilidad del manejo y por proporcionar una fuente de energía limpia y renovable al producir biogás ${ }^{11}$. Las ventajas de un reactor anaerobio son la baja producción de residuos sólidos biológicos (lodos), bajo requerimiento de nutrientes, el metano es un producto final muy útil, genera energía y es posible una carga orgánica elevada; las desventajas son el crecimiento lento de microorganismos, malos olores, requerimientos más elevados de regulador para controlar el $\mathrm{pH}$ y escasa eficiencia de eliminación de residuos sólidos ${ }^{10}$. 
La digestión anaerobia es un proceso microbiológico muy complejo, que tiene lugar en el seno de una fase acuosa y mediante el cual la materia orgánica (MO) se transforma, principalmente, en biogás $(\mathrm{CO} 2+\mathrm{CH} 4)$ y nuevos microorganismos (lodo) ${ }^{12}$. La digestión anaeróbica está conformada por cuatro etapas metabólicas: hidrólisis, acidogénesis, acetogénesis y metanogénesis. Un número diverso de bacterias participan en las tres primeras etapas y en la formación de metano (por arqueas metanogénicas) que deriva principalmente de acetato e hidrógeno (el $\mathrm{CO}_{2}$ viene a ser un subproducto). La digestión anaerobia de lodo residual sigue siendo limitada debido a la hidrólisis, considerada el paso limitante de la velocidad de todo el proceso y con el fin de aumentar o acelerar las tasas de biodegradación, se ha probado diversos tratamientos previos como la hidrólisis térmica, enzimática, química o mecánica ${ }^{13}$.

En los tratamientos de doble fase (termófilo/mesófilo), la etapa de la acidogénesis termofílica ofrece beneficios operacionales, como el aumento de las tasas de hidrólisis, producción de Ácidos Grasos Volátiles (AGVs) y la reducción del volumen del digestor. La información de este tipo de tratamiento es limitada, de lo recopilado, la etapa acidogénica termófila se optimiza para alcanzar la tasa máxima de producción de ácido acético y regularmente como un tratamiento complementario, es conectado a un reactor anaerobio metanogénico mesófilo para terminar el tratamiento. Existe la controversia si la acidificación completa o parcial mejoraría la eficiencia del tratamiento anaerobio. Sin embargo, en los procesos de doble fase donde se acidifican completamente aguas residuales, pueden generar efectos sobre la construcción y costos operativos, debido a la necesidad de acoplar un reactor adicional para la acidificación completa de los compuestos orgánicos influyentes ${ }^{14}$. La mayoría de estos estudios se han enfocado en la etapa de acidogénesis de reactores mesófilo, mientras que en los termófilos las investigaciones son muy limitadas ${ }^{15}$.

Este trabajo tiene como objetivo evaluar el comportamiento en tres momentos de tratamiento M1 (1 - 418 días), M2 (419 - 641 días) y M3 (420 - 1001 días), a diferentes Tiempos de Retención Hidráulicas (TRH) y caudales en un Reactor Anaerobio Termófilo Metanogénico, todo esto para brindar un tratamiento para un lodo mixto (mezcla de lodo de un decantador primario y secundario) procedente de la Estación Depuradora de Aguas Residuales (EDAR) de Valladolid - España.

\section{PARTE EXPERIMENTAL}

A. Lodo a tratar: La Universidad de Valladolid (UVA) cuenta con un convenio con la Estación Depuradora de Aguas Residuales de Valladolid (EDAR), entidad que proporcionó el lodo para la investigación, el mismo que fue colectado de la etapa de espesamiento del decantador primario y secundario, a esta mezcla se le denominó lodo mixto espesado.

B. Reactor: Para esta investigación se trabajó con un reactor termófilo $55 \mathrm{C}^{\circ}( \pm 2)$ de 50 litros de capacidad, dicho reactor ya se encontraba en funcionamiento intermitente por lo que no fue necesario inocularlo. 
Los componentes del sistema de tratamiento fueron: un tanque homogenizador conectado a una bomba de regulación de caudal de alimentación, un tanque de almacenamiento de lodo tratado, un compresor para la reinyección del biogás para agitar el lodo y así mejorar la superficie de contacto, un condensador para la separación del vapor de agua del biogás, una bomba de recirculación de lodo, un equipo de pulsos para determinar la producción de biogás producido y válvulas para toma de muestras. A continuación, se muestra el esquema del reactor en la figura 1.

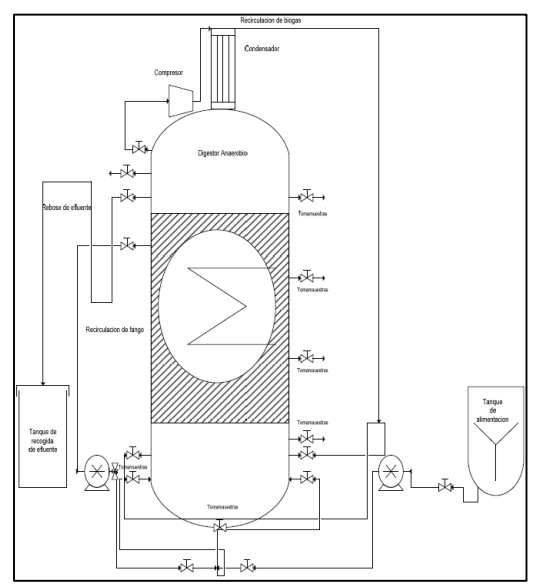

Figura 1. Esquema de un Reactor Termófilo Anaerobio Fuente: Benito J. 2011.

C. Monitoreo de parámetros: Se tomaron muestras en la alimentación y salida del tratamiento, se realizaron análisis en laboratorio de parámetros como: Demanda Química de Oxígeno total (DQOt), Sólidos Totales y Volátiles (ST y SV), Amonio, Nitrógeno Kjeldahl (NKT); la Centrifugabilidad, Filtrabilidad, Tiempo de Succión Capilar (CST), Viscosidad, Ácidos Grasos Volátiles (AGVs) y la Alcalinidad, todos estos parámetros antes mencionados fueron analizados tomando como referencia el Standard Methods ${ }^{16}$. Se determinó la composición con un equipo de cromatografía de gases y con un equipo de pulsos el volumen del biogás. El cálculo de los parámetros control se muestra en la tabla 1 . 
Tabla 1. Fórmulas de Parámetros de Control del Reactor Termófilo.

\begin{tabular}{cc}
\hline Parámetro & Fórmula \\
\hline Carga Másica & $\mathrm{CM}=\frac{\mathrm{g} \mathrm{SV}_{\text {alimentación }}}{\mathrm{gSV}_{\text {reactor }} \times \text { día }}$ \\
Tiempo de Retención Hidráulica & $\mathrm{TRH}=\frac{\text { Litros }_{\text {reactor }}}{\text { Caudal }_{\text {alimentación }}}$ \\
Productividad de biogás & Productividad $=\frac{\mathrm{ml}_{\text {biogás }}}{\mathrm{g} \mathrm{SV}_{\text {eliminada }}}$ \\
Carga volumétrica & $\mathrm{CV}=\frac{\mathrm{g} \mathrm{SV}_{\text {alimentación }}}{\text { Litros }_{\text {reactor }} \times \text { día }}$
\end{tabular}

Fuente: Elaboración propia

D. Estrategia de tratamiento: Consistió en evaluar los efectos del reactor sometiéndolo a estrés, reduciendo los Tiempos de Retención Hidráulicas (TRH) en tres momentos, el primero y el segundo fue de 22,52 a 11,26 días y el tercero de 22,52 a 4,50 días. Al someter a estrés al reactor se evaluó los cambios en los parámetros monitoreados, analizados en laboratorio y descritos en el acápite " $\mathrm{C}$ ".

\section{RESULTADOS Y DISCUSIÓN}

Algunos estudios se centran en la influencia del TRH en la sedimentabilidad de los fangos entre otros parámetros también de interés, evaluando fases anaerobias en seis ensayos con diferentes TRH y distintos tiempos de reacción anaerobia ${ }^{7}$. En esta investigación se evaluó el desempeño del reactor, fue afectado cada vez que se reducía el Tiempo de Retención Hidráulica (TRH), el criterio que se tomó para la disminución fue la estabilidad en los parámetros, como la concentración de amonio, productividad de biogás, $\%$ eliminación de DQO, ST y SV. Las condiciones operacionales de alimentación promedio en función del TRH en los tres momentos se muestran en la tabla 2. 
Tabla 2. Evolución de los parámetros de alimentación a diferente TRH.

\begin{tabular}{|c|c|c|c|c|c|c|c|c|c|}
\hline \multirow[b]{2}{*}{ Días } & \multirow[b]{2}{*}{ Mto. } & \multicolumn{8}{|c|}{ Parámetros } \\
\hline & & $\begin{array}{l}\text { DQO } \\
(\mathrm{mg} / \mathrm{L})\end{array}$ & pH & $\begin{array}{c}\text { ST } \\
(\mathrm{g} / \mathrm{L})\end{array}$ & $\begin{array}{c}\text { SV } \\
(\mathrm{g} / \mathrm{L})\end{array}$ & $\begin{array}{c}\mathrm{NH}_{3}^{+} \\
(\mathrm{mg} / \mathrm{L})\end{array}$ & $\begin{array}{c}\text { NKT } \\
(\mathrm{mg} / \mathrm{Kg})\end{array}$ & $\begin{array}{c}\mathrm{CV} \\
(\mathrm{gSV} / \mathrm{L} \cdot \mathrm{d})\end{array}$ & $\begin{array}{c}\mathrm{CM} \\
(\mathrm{gSV} / \mathrm{gSV} \cdot \mathrm{d})\end{array}$ \\
\hline \multirow{3}{*}{22,5} & M1 & 53339 & 8,24 & 47,0 & 26,8 & 829,9 & 2343,2 & 0,66 & 0,01 \\
\hline & M2 & 33186 & 8,13 & 14,2 & 10,9 & 328,2 & 1421,7 & 0,40 & 0,01 \\
\hline & M3 & 33733 & 8,18 & 26,1 & 17,4 & 432,7 & 1817,3 & 0,62 & 0,01 \\
\hline \multirow{3}{*}{15,03} & M1 & 42535 & 8,15 & 27,2 & 18,1 & 406,8 & 1990,2 & 1,17 & 0,03 \\
\hline & M2 & 34436 & 7,83 & 25,4 & 19,7 & 325,4 & 1395,4 & 1,12 & 0,03 \\
\hline & M3 & 27594 & 8,10 & 24,2 & 18,7 & 314,0 & 2012,4 & 0,95 & 0,02 \\
\hline \multirow{3}{*}{11,26} & M1 & 34097 & 8,09 & 25,2 & 16,1 & 259,2 & 1511,9 & 1,19 & 0,02 \\
\hline & M2 & 32453 & 8,13 & 24,9 & 17,9 & 306,1 & 1734,1 & 1,24 & 0,03 \\
\hline & M3 & 26863 & 7,96 & 26,6 & 21,0 & 218,8 & 1729,5 & 1,46 & 0,03 \\
\hline 7,5 & M3 & 29433 & 7,88 & 24,4 & 18,9 & 190,5 & 1763,7 & 2,09 & 0,04 \\
\hline 5,6 & M3 & 30692 & 7,90 & 32,5 & 23,7 & 204,0 & 2046,8 & 3,29 & 0,06 \\
\hline 4,5 & M3 & 31386 & 7,92 & 33,5 & 21,8 & 234,5 & 1757,0 & 3,86 & 0,07 \\
\hline
\end{tabular}

Fuente: Elaboración propia

En la figura 2 se presenta el TRH, el cual se modificó por el aumento del caudal de alimentación en cada momento. Se trabajó con dos tipos de TRH, el real y el teórico (programación de la bomba de alimentación), si bien se observa que los valores reales frente a los teóricos fueron mayores, ambos describen un comportamiento muy similar. También se observa que existe mayor variabilidad en los TRH reales, esto se debió a la consistencia del lodo.

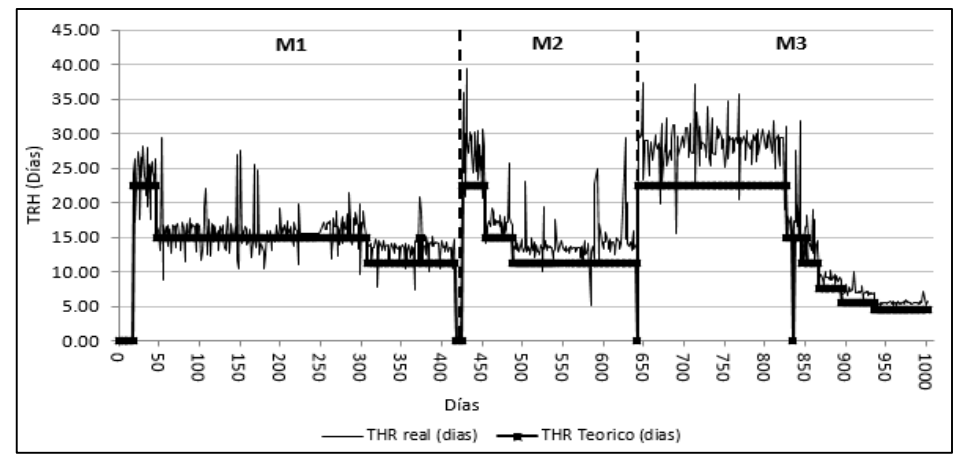

Figura 2. Evolución del Tiempo de Retención Hidráulica Fuente: Elaboración propia.

En general, a mayor concentración de sólidos volátiles a la entrada, mayor concentración de sólidos a la salida. Sin embargo, a pesar de tener una concentración menor de sólidos volátiles, la eliminación es mayor ${ }^{2}$. De acuerdo a la figura 3, los datos experimentales obtenidos de los Sólidos Volátiles (SV) de la entrada y la salida fueron variables, esto influenció al TRH real (la bomba de alimentación suministraba diferentes concentraciones de SV al reactor). Se observa ciertos días donde los valores de SV entrada son menores a los de la salida, esto se debe a que el lodo tenía menos consistencia ciertas semanas que otras. 


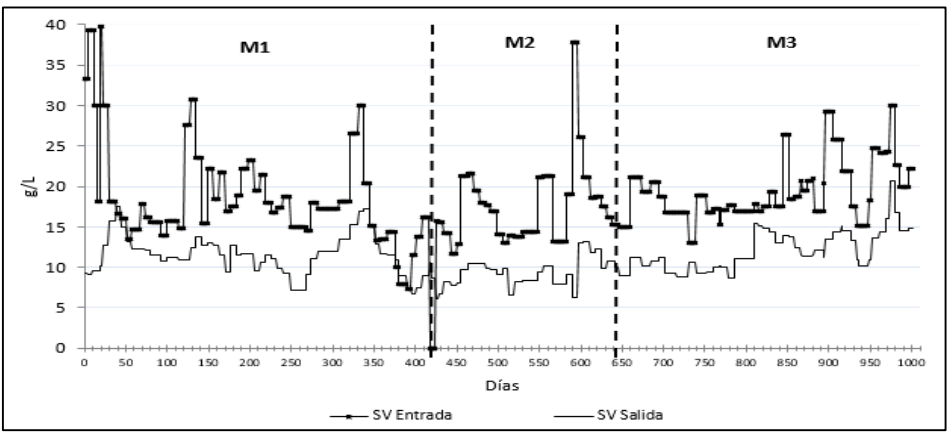

Figura 3. Evolución de los Sólidos Volátiles a la entrada y salida del tratamiento.

Fuente: Elaboración propia.

A 6 días de TRH y menor carga orgánica en su etapa VI, se observó una disminución sobre la eficiencia de eliminación de DQO $(40,6 \%)^{18}$. En esta investigación, en la figura 4, se muestra el comportamiento del \% de eliminación de la Demanda Química de Oxígeno (DQO), por cada momento, se observa que fue muy variable, esto se debería por la concentración de los SV que tiene un comportamiento similar y la disminución del TRH, pero logra estabilizarse e incrementar el \% de eliminación de la DQO, que en promedio fue de $42 \%$. Los valores de la DQO de entrada y salida también describen un comportamiento irregular teniendo un valor promedio de 35982 y $19496 \mathrm{mg} / \mathrm{L}$, respectivamente.

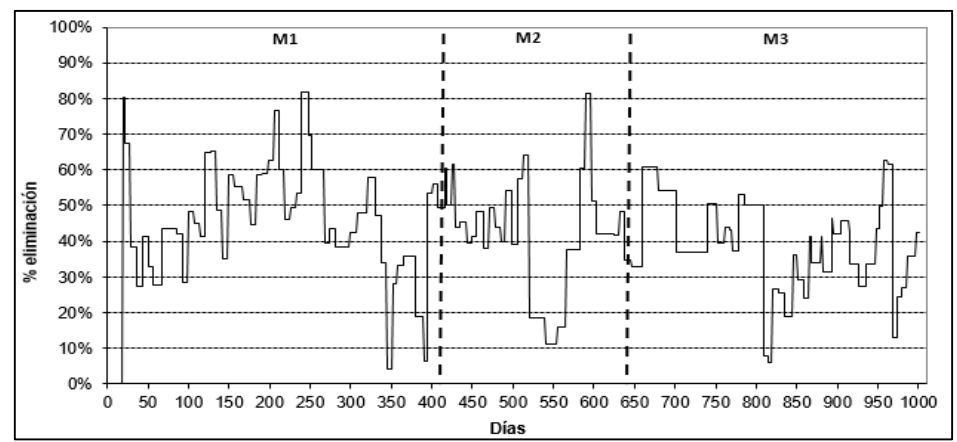

Figura 4. Porcentaje de eliminación de la DQO. Fuente: Elaboración propia.

La mayor acumulación de AGVs, genera inestabilidad en el reactor, por posibles problemas de acidificación si no se controla adecuadamente. Por esta razón es necesario un control minucioso de los caudales de fases, para evitar este tipo de problemas ${ }^{2}$. La evolución de los Ácidos Grasos Volátiles (AGVs), se evaluaron con dos métodos, el primero con un equipo de 
cromatografía el cual generaba valores más exactos, pero demoraban 5 días, el otro método fue por valoración siendo el menos exacto pero el más inmediato (una hora), en la figura $5 \mathrm{se}$ puede observar que los valores fueron muy cercanos y de comportamiento similar. Al inicio del momento "M1" el reactor estaba en proceso de adecuación, por ello existía una mayor diferencia entre los métodos de análisis.

En el momento "M3", en el día 965 aproximadamente, se observa un incremento en la concentración de los ácidos grasos, esto se debió al aumento del caudal y por llevar al reactor a un punto de estrés, pero luego de unos días se estabiliza y tolera estos cambios.

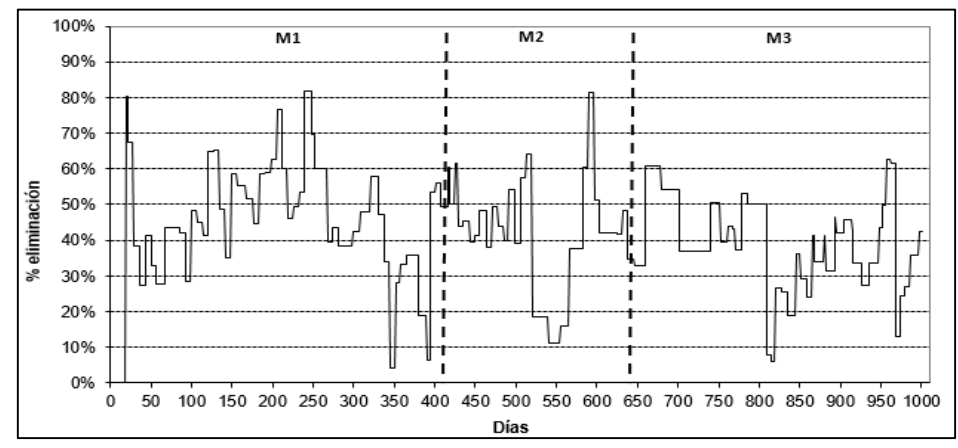

Figura 5. Concentración del AGV del método de valoración y cromatografía. Fuente: Elaboración propia.

A medida que pasa el tiempo aumenta la concentración de sólidos en el reactor, aunque la concentración de sólidos en la alimentación sea mayor, el reactor amortigua esa concentración, y la carga másica resulta ser menor que en las primeras etapas de tratamientos ${ }^{2}$. En esta investigación se muestra, en la figura 6, el comportamiento que describió los valores de la productividad de biogás, con la Carga Másica (CM) fue inversamente proporcional. Se observa valores picos de la productividad, esto se debió a problemas con la alimentación (obstrucciones) se puede tomar como datos “outlier". Además, en la producción de biogás el porcentaje promedio de metano en "M1; M2 y M3" fue 62,4031; 61,2108 y 62,1083 \%, respectivamente. La CM tuvo un comportamiento irregular que se debió principalmente a la concentración variable de sólidos en el lodo. En el momento 3 se observa el incremento de la carga másica, esto se debe al aumento del caudal o reducción del TRH, pero la productividad de biogás se mantuvo.

En su etapa VI se observó un incremento en la producción de biogás predominando el $\mathrm{CH} 4$ en un 68,2 \% y disminuyendo el CO2 (31,8\%), al favorecerse el desarrollo de las condiciones metanogénicas $(\mathrm{pH}>7,1)$, debido al aumento del TRH10. Muy similar al de esta investigación. 


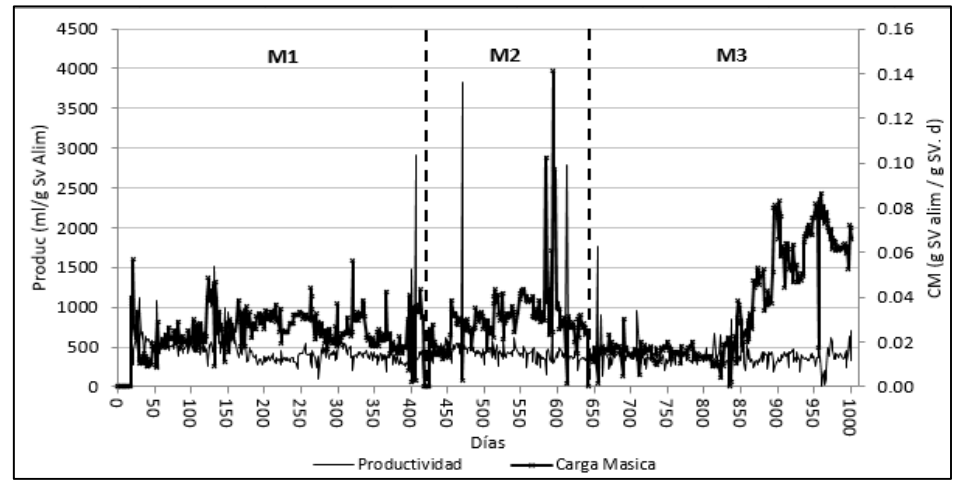

Figura 6. Productividad vs Carga Másica. Fuente: Elaboración propia.

Carga Másica (CM) y el \% eliminación de SV tienen un comportamiento similar a diferencia del "M3", donde el \% de eliminación disminuye, esto es un indicativo de que el reactor está siendo sometido a estrés (figura 7).

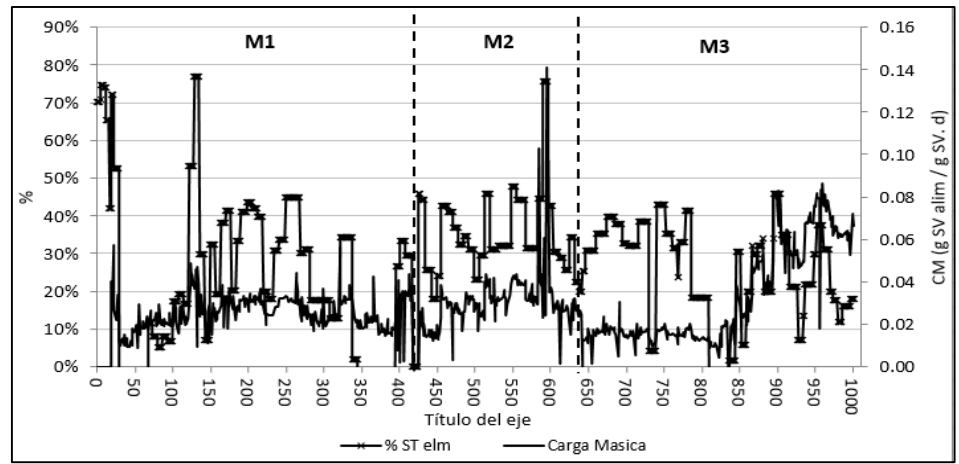

Figura 7. \% Eliminación SV vs Carga Másica.

Fuente: Elaboración propia.

Los g SV alimentados que se convirtieron a $\mathrm{ml}$ de biogás (productividad) y la cantidad de biogás que se generó tuvo un comportamiento ascendente debido al incremento de la CM. En la generación de metano en "M1, M2 y M3" en promedio fueron 30, 27 y 40 L/d, respectivamente, en el momento "M3" se observa un incremento porque las bacterias tenían más concentrado, por ende mayor biogás.

Se realizaron en el momento 3 pruebas complementarias como CST, Deshidratabilidad, Filtrabilidad y Viscosidad, en laboratorio a la entrada y salida del reactor en el 879 y 915 días, en los cuales sufrieron una disminución del TRH teórico de 7,5 a 5,6 días. 
Para medir la filtrabilidad se realizó con la prueba del Tiempo de Succión Capilar (CST), utilizó la presión de succión capilar en un papel poroso en el tiempo, filtrado con el avance entre los electrodos separados radialmente. La velocidad del filtrado pasa por un medio filtrante y es controlada por la filtrabilidad de la suspensión, de tal modo que a menor valor de CST, la filtrabilidad de un lodo será mejor. La filtrabilidad no es afectada significativamente por la disminución del TRH teórico de 7,5 a 5,6 días.

La separación en una fase sólida y líquida del lodo se realiza por el método de la centrifugación, se da por la sedimentación del sólido con partículas de mayor densidad. La centrifugabilidad en los días 879 y 915 en la alimentación como la salida son muy similares para el \% de recuperación de la torta y la concentración de sólidos en la torta.

La viscosidad se define como la medida de la fricción interna de un fluido en relación con otro. En los días 879 y 915 se analizó la viscosidad del lodo en la entrada $(0,2529$ y 0,2525$)$ y salida $(0,1786$ y 0,1812$)$ centi poise $(\mathrm{cP})$, respectivamente, en ambos casos después del tratamiento, la viscosidad descendió.

\section{CONCLUSIONES}

- Por más que se incrementó el caudal en los tres momentos de evaluación, el reactor metanogénico no llegó a transformarse en un acidogénico, porque continuó produciendo biogás con un porcentaje de promedio de metano de $62,0825 \%$ y la eliminación de la DQO en promedio fue de $42 \%$.

- En los momentos del tratamiento y en particular en el "M3", se observa incremento de la carga másica por el incremento del caudal o reducción del TRH, pero manteniéndose la productividad de biogás.

- En los tres momentos sujetos a variaciones en los TRH, el reactor demostró ser estable al asimilar los cambios en la composición y características del lodo de alimentación.

- Durante el tratamiento anaerobio termófilo la centrifugabilidad y filtrabilidad del lodo no varía significativamente.

- $\quad$ No se detectaron problemas de inhibición de ácidos grasos porque en los tres momentos del tratamiento los resultados de los AGVs no superaron los $2181 \mathrm{mg} \mathrm{AcOH} / \mathrm{L}$.

\section{AGRADECIMIENTOS}

Agradezco a todas las personas que colaboraron con el desarrollo de este trabajo de investigación, en particular a mi hermana Diana Morales, mi padre Javier Morales, a mi madre Norma Chuquimantari, hermanita Nataly Morales, a mi asesor Fernando Fernández Polanco y a un gran amigo Jaime Benito, quienes hicieron posible cumplir este objetivo. 


\section{REFERENCIAS BIBLIOGRÁFICAS}

1. Crisanto C. Evaluación de estiércol de equino y aguas residuales domésticas para la producción de biogás y biol mediante digestores semi continuos. [Tesis de pregrado]. Lima, Perú: Universidad Nacional Agraria La Molina; 2016.

2. Benito J. Variación de las condiciones operacionales habituales de los digestores anaerobios de lodos: Digestión Anaerobia Termófila. [Tesis de Master]. Valladolid -España: Universidad de Valladolid; 2011.

3. Delgado R. Tratamiento de efluentes de un reactor uasb mediante una mezcla de amonio cuaternario y hierro. [Tesis de pregrado]. Lima, Perú: Universidad Nacional Agraria La Molina. Perú; 2018.

4. Lahera V. Infraestructura Sustentable: Las plantas de tratamiento de aguas residuales. Madrid, España: Universidad Autónoma; 2010.

5. MINAM. D.S N 003-2010 MINAM, Límites Máximos Permisibles para los efluentes de Plantas de Tratamiento de Aguas Residuales Domésticas o Municipales. El Peruano. 2010 Mar 10: 415675-415676.

6. Ley 22/2011 de Residuos y Suelos Contaminados, España. BOE [Internet]. 2011 [accesado 18 jul 2019]. Disponible en: https:/www.boe.es/buscar/pdf/2011/BOE-A2011-13046-consolidado.pdf

7. Real Decreto 1310/1990, Utilización de lodos de depuración en el sector agrario, 1990. España. BOE [Internet]. 1990; 262: 32339-32340 [accesado 17 jul 2019]. Disponible en: https:/www.boe.es/boe/dias/1990/11/01/pdfs/A32339-32340.pdf

8. Directiva 86/278/CEE, Protección del medio ambiente y, en particular, de los suelos, en la utilización de los lodos de depuradora en agricultura. Comunidad Económica Europea. Diario Oficial de las Comunidades Europeas [Internet]. 1986; 4. 7. 86: N L 181/6-L 181/12 [accesado 17 jun 2019]. Disponible en: https://eur-lex.europa.eu/legalcontent/ES/TXT/PDF/?uri=CELEX:31986L0278\&from=ES

9. DL N 1278 MINAM, Ley De Gestión Integral de Residuos Sólidos, Perú. [Internet]. 2017 [Accesado 02 nov 2019]. Disponible en: http://www.minam.gob.pe/wp-content/ uploads/2017/04/Decreto-Legislativo-N\%C2\%B0-1278.pdf

10. McCarty P, Rittmann B. Biotecnología del Medio Ambiente. México DF: Editorial Mc Graw Hill; 2001.

11. Sáez J. Diseño de un reactor anaerobio para la digestión de fangos. [Tesis de grado de Ingeniero] Valencia: Universidad de Valencia - España; 2017. [Accesado el 01 agosto del 2019]. Disponible en: https://riunet.upv.es/bitstream/handle/10251/100168/ S\%C3\%81EZ\%20-\%20Dise\%C3\%B10\%20de\%20un\%20reactor\%20anaerobio\%20 para $\% 201 \mathrm{a} \% 20$ digesti $\% \mathrm{C} 3 \% \mathrm{~B} 3 \mathrm{n} \% 20 \mathrm{de} \% 20$ fangos.pdf? sequence $=1$

12. Mendes L. Estudio de un sistema híbrido uasb + mbr para el tratamiento de aguas residuales urbanas [Tesis de Maestria]. Alicante: Universidad de Alicante, España; 2015 [Accesado el 15 de julio 2019] Disponible en: https://iuaca.ua.es/es/master-agua/ documentos/-gestadm/trabajos-fin-de-master/tfm09/tfm09-lyvia-mendes-predolin.pdf

13. Gagliano MC, Braguglia CM, Gallipoli A, Gianico A, Rossetti S. Microbial diversity in innovative mesophilic/thermophilic temperature-phased anaerobic digestion of sludge. Environ Sci Pollut Res Int. 2015;22(10):7339-7348. 
14. Kim W, Shin SG, Cho K, Lee C, Hwang S. Performance of methanogenic reactors in temperature phased two-stage anaerobic digestion of swine wastewater. J Biosci Bioeng. 2012;114(6):635-639.

15. Yu H-Q, Fang H, Gu G-W, Comparative performance of mesophilic and thermophilic acidogenic upflow reactors. Process Biochem. 2002; 38: 447-454.

16. Standard Methods APHA. Standard methods for the examination of water and wastewater. 22nd ed. Washington, DC, EEUU: American Public Health Association; 2005.

17. Muda K, Aris A, Salim M, Ibrahim Z, van Loosdrecht M, Ahmad A, et al. The effect of hydraulic retention time on granular sludge biomass in treating textile wastewater. Water Res. 2010; 45(16): 4711-472.1

18. Terreros-Mecalco J, Olmos-Dichara A, Noyola-Robles A, Ramírez-Vives F y MonroyHermosillo O. Digestión anaerobia de lodo primario y secundario en dos reactores UASB en serie. Rev Mex Ing Quím. 2009; 8(2): 153-161. 